\title{
Magnetohydrostatic modeling of AR11768 based on a SunRISE/IMaX vector magnetogram
}

\author{
X. Zhu (朱小帅) ${ }^{1}$, T. Wiegelmann ${ }^{1}$, and S K. Solanki ${ }^{1,2}$ \\ ${ }^{1}$ Max-Planck-Institut für Sonnensystemforschung, Justus-von-Liebig-Weg 3, 37077 Göttingen, Germany \\ e-mail: zhu@mps .mpg. de \\ 2 School of Space Research, Kyung Hee University, Yongin, Gyeonggi 446-701, Republic of Korea \\ Received 18 February 2020 / Accepted 28 May 2020
}

\begin{abstract}
Context. High-resolution magnetic field measurements are routinely only done in the solar photosphere. Higher layers, such as the chromosphere and corona, can be modeled by extrapolating these photospheric magnetic field vectors upward. In the solar corona, plasma forces can be neglected and the Lorentz force vanishes. This is not the case in the upper photosphere and chromosphere where magnetic and nonmagnetic forces are equally important. One way to deal with this problem is to compute the plasma and magnetic field self-consistently, in lowest order with a magnetohydrostatic (MHS) model. The non-force-free layer is rather thin and MHS models require high-resolution photospheric magnetic field measurements as the lower boundary condition.

Aims. We aim to derive the magnetic field, plasma pressure, and density of AR11768 by applying the newly developed extrapolation technique to the SUNRISE/IMaX data embedded in SDO/HMI magnetogram.

Methods. We used an optimization method for the MHS modeling. The initial conditions consist of a nonlinear force-free field (NLFFF) and a gravity-stratified atmosphere. During the optimization procedure, the magnetic field, plasma pressure, and density are computed self-consistently.

Results. In the non-force-free layer, which is spatially resolved by the new code, Lorentz forces are effectively balanced by the gas pressure gradient force and gravity force. The pressure and density are depleted in strong field regions, which is consistent with observations. Denser plasma, however, is also observed at some parts of the active region edges. In the chromosphere, the fibril-like plasma structures trace the magnetic field nicely. Bright points in SUNRISE/SuFI $3000 \AA$ A images are often accompanied by the plasma pressure and electric current concentrations. In addition, the average of angle between MHS field lines and the selected chromospheric fibrils is $11.8^{\circ}$, which is smaller than those computed from the NLFFF model $\left(15.7^{\circ}\right)$ and linear MHS model $\left(20.9^{\circ}\right)$. This indicates that the MHS solution provides a better representation of the magnetic field in the chromosphere.
\end{abstract}

Key words. Sun: magnetic fields - Sun: chromosphere - Sun: photosphere

\section{Introduction}

Nonmagnetic forces should be taken into account when we study the photosphere and chromosphere owing to the high plasma $\beta$ in these layers. If we neglect dynamics and plasma flow, then the resulting static state of the system can be described by the so-called magnetohydrostatic (MHS) assumption, which is determined by the balance of Lorentz force, pressure gradient, and gravity force, together with the solenoidal condition of the magnetic field.

The 3D MHS equations can be linearized by some assumptions. Analytical solutions for the linear MHS (LMHS) model have been developed in many papers (e.g., Low 1985, 1991; Osherovich 1985; Neukirch 1997; Petrie \& Neukirch 2000; Neukirch \& Wiegelmann 2019). These solutions have been used for a number of specific applications to the Sun (e.g., Bagenal \& Gibson 1991; Zhao \& Hoeksema 1993; Aulanier et al. 1998, 1999; Petrie 2000; Ruan et al. 2008; Gent et al. 2013; Wiegelmann et al. 2015, 2017) and stars (Lanza 2008; MacTaggart et al. 2016).

A more challenging problem is to solve the MHS equations in the generic case, which can only be done with a numerical approach. Several methods have been developed to solve these equations. The magnetohydrodynamic (MHD) relaxation method was introduced to derive the MHS solution by using an "evolution technique" (McClymont \& Mikic 1994; Jiao et al. 1997; Zhu et al. 2013, 2016; Miyoshi et al. 2020). The Grad-Rubin iteration, which is well known in the calculation of the nonlinear force-free field (NLFFF), was extended by Gilchrist \& Wheatland (2013) and Gilchrist et al. (2016) to solve the MHS equations. Wiegelmann \& Inhester (2003), Wiegelmann \& Neukirch (2006) and Wiegelmann et al. (2007) developed the optimization method to treat the MHS equations. We recently extended this method by introducing the gravity force (Zhu \& Wiegelmann 2018). In addition, our new algorithm ensures that the resulting plasma pressure and density are positive definite. More recently, we further tested our code with the radiative MHD simulation of a solar flare (Zhu \& Wiegelmann 2019). This challenging test, in which solar flares are intrinsically non-static and even nonstationary, provides a solid foundation for the application of the code to real observations.

It is worth noting that, besides the traditional NLFFF models, a new type of NLFFF model (Malanushenko et al. 2012; Aschwanden 2013, 2016) has recently been introduced. This alternative approach uses the line-of-sight magnetogram to constrain the potential field. With the help of a forwardfitting method, the angle between the magnetic field and coronal or chromospheric loops is minimized. In this model, the 

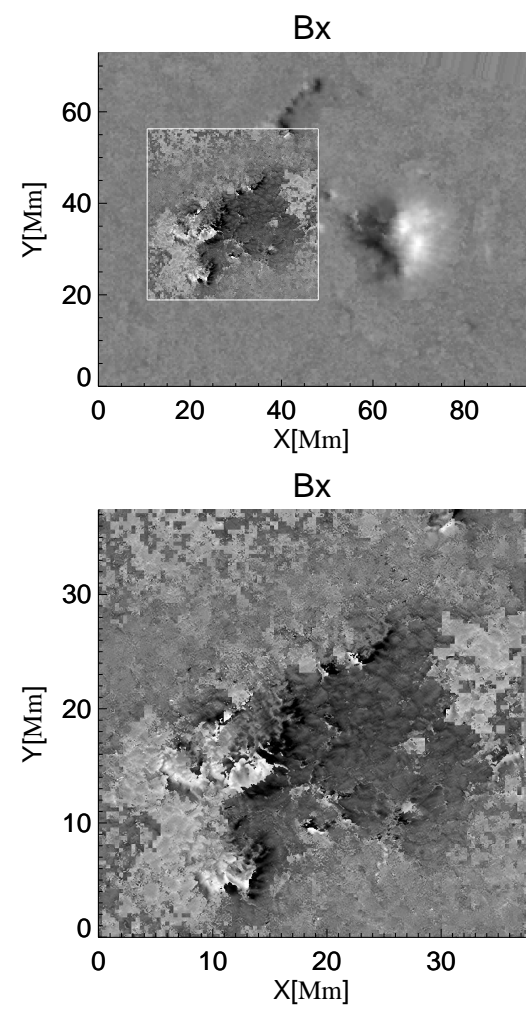

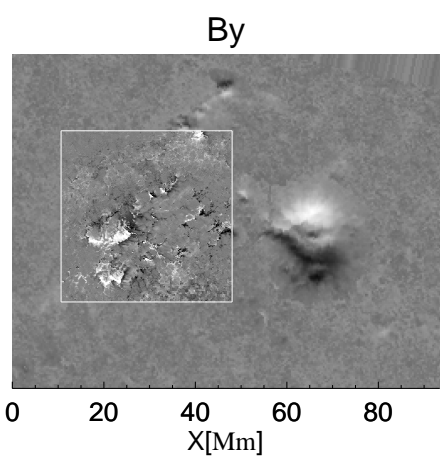

By

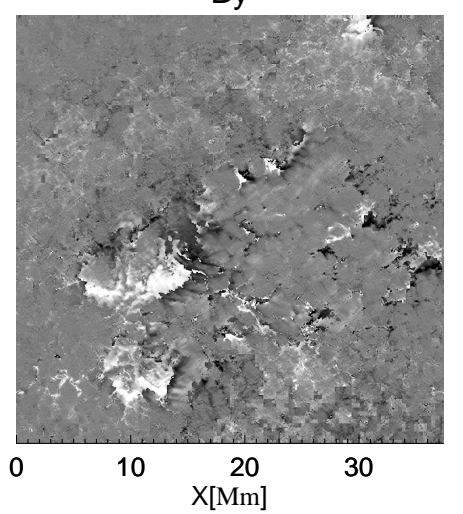

$\mathrm{Bz}$

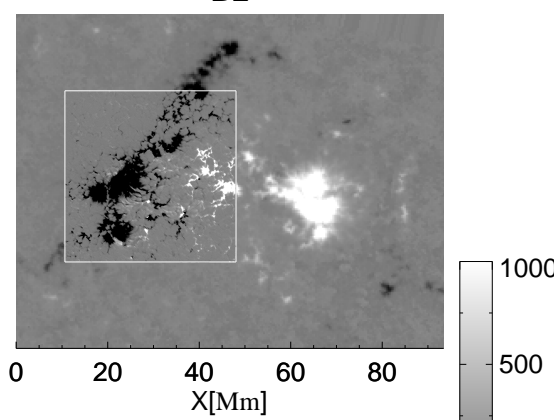

$\mathrm{Bz}$

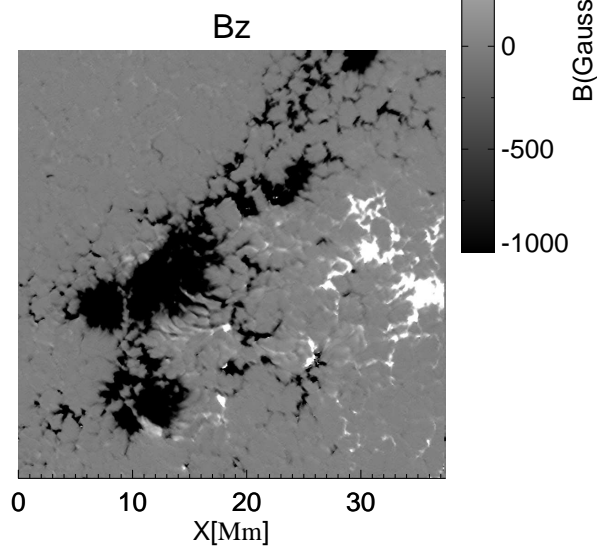

Fig. 1. Top: IMaX measurements at 23:48 UT embedded in the vector magnetogram of HMI (observed at 23:48 UT). The outlined region with a clearly visible higher resolution is the IMaX FOV. Bottom: vector magnetogram of IMaX.

assumption of a force-free photosphere is not used either. This works well in the corona, but may not be the ideal way to describe the non-force-free chromospheric field.

In this paper, we apply our code, for the first time, to a vector magnetogram obtained by the Imaging Magnetograph eXperiment (IMaX) instrument on the SUNRISE balloon-borne solar observatory. Since the field of view (FOV) of IMaX is limited to part of the active region, an Helioseismic and Magnetic Imager (HMI) vector magnetogram is used to cover the unobserved parts. The organization of the paper is as follows. In Sect. 2, we describe the dataset used in this paper. In Sect. 3, we analyze the results and compare these results with other models. Conclusions and perspectives are presented in Sect. 4.

\section{Magnetohydrostatic extrapolation and its application to AR11768}

The MHS extrapolation computes the magnetic field, plasma pressure, and density consistently with the help of an optimization principle. The algorithm was described and tested in detail in Zhu \& Wiegelmann $(2018,2019)$.

The primary dataset used in this work was recorded by the vector magnetograph IMaX (Martínez Pillet et al. 2011) on board the SunRISE balloon-borne solar observatory (Barthol et al. 2011; Berkefeld et al. 2011; Gandorfer et al. 2011) during its second flight, referred to as SUNRISE II (Solanki et al. 2017). The IMaX data have a pixel size of $40 \mathrm{~km}$ a FOV that contains $936 \times 936$ pixels $\left(50^{\prime \prime} \times 50^{\prime \prime}\right)$. This FOV is limited to part of AR11768. The data were inverted by Kahil et al. (2017) via the SPINOR inversion code (Frutiger et al. 2000), which builds on STOPRO routines (Solanki 1987). A one-component atmospheric model with three optical depth nodes (at $\log \tau=-2.5$,
$-0.9,0)$ for the temperature and height-independent magnetic field is applied. The effect of the inversion on the extrapolation result was studied by Wiegelmann et al. (2010) and found to be minor; the latter authors used a different inversion than we used in this work. The $180^{\circ}$ ambiguity is removed with an acute angle method (Metcalf et al. 2006) that minimizes the angle with, in this case, the corresponding HMI vector magnetogram. We note that there is a square pattern in the transverse field of IMaX (Fig. 1). This originates from disambiguating IMaX with HMI data in which a square patten also exists (seen in the transverse field). Essentially, the square pattern is the IMaX noise that is modulated to match the HMI spatial resolution by disambiguating. Although this noise appears in big mosaics (i.e., big compared to the IMaX resolution), the size of every single mosaic is still very small compared to the active region. Therefore the effect they have on the extrapolation is expected to be similar to the effect of the normal noise that has been studied by Zhu \& Wiegelmann (2018). In that paper, based on the quantitative assessment for the extrapolation, we found the influence of the random noise ( $20 \%$ level) on the magnetic field to be less than $4 \%$. As pointed out by De Rosa et al. (2009), it is necessary to have flux balance within the FOV and to catch the magnetic connectivity to find unique solutions. Thus we embedded the IMaX data into the HMI vector magnetogram (Pesnell et al. 2012; Scherrer et al. 2012) taken closest in time to the analyzed IMaX magnetogram. Figure 1 shows both IMaX and combined vector magnetogram.

Figure 2 depicts the flow chart of our code applied to the combined magnetogram. The following steps are plotted. First, we compute a NLFFF (Wiegelmann 2004) with a preprocessed magnetogram (Wiegelmann et al. 2006) and a gravitystratified atmosphere along field lines with a 1D temperature profile (Fig. 3); the net Lorentz force and torque are removed within the error margin of the measurement using a minimization 


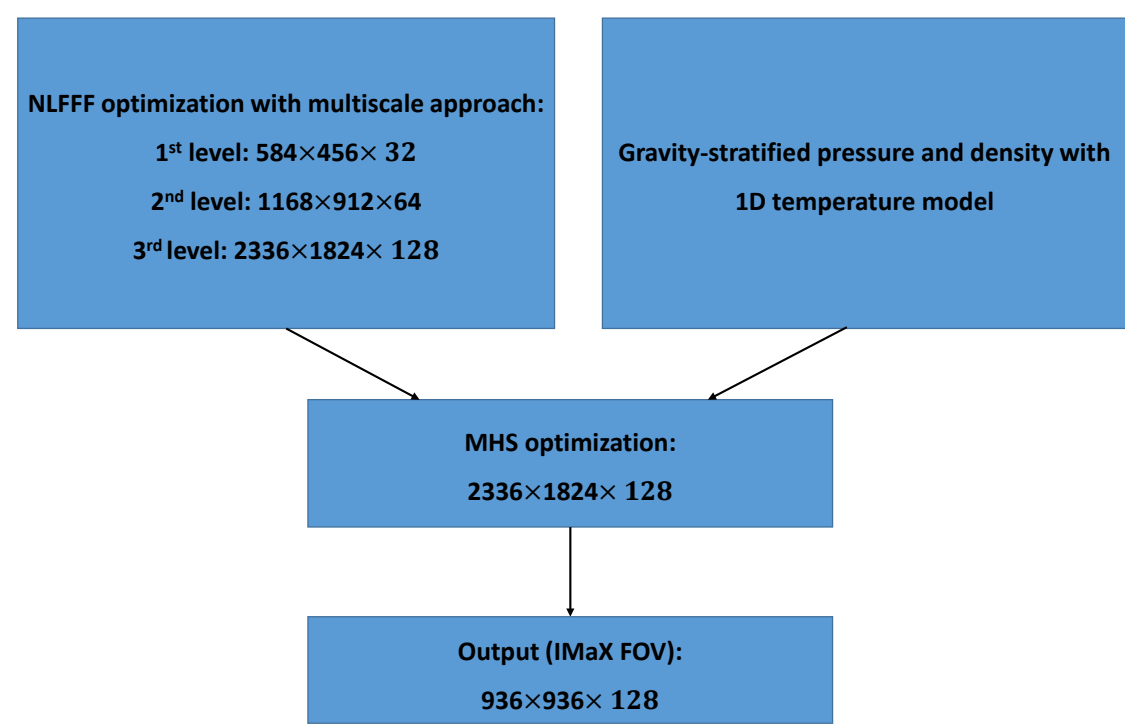

Fig. 2. Schematic flow chart of the MHS code applied to a vector magnetogram. principle to make the data compatible with the force-free assumption. The pressure on the bottom boundary is computed using $p=p_{\text {quiet }}-\frac{1}{2} B_{z}{ }^{2}$, where $p_{\text {quiet }}$ is the pressure in the quiet region. The bottom density is determined by assuming a uniform temperature of 6000 Kelvin on the photosphere. Second, we carry out a further optimization to achieve an MHS equilibrium with the original magnetogram. We note that although a temperature profile is given at the initial state to relate the gas pressure and density, the initial temperature profile is no longer a restriction on the pressure and density optimization. There are two options to input the bottom magnetic boundary. The option used in this study replaces the preprocessed magnetogram with the original magnetogram directly. The other option involves changing the magnetic field gradually from the preprocessed value to the original value. The side and top boundaries are fixed to their initial values, which are potential fields. The computation is performed in a $2336 \times 1824 \times 128$ box with a $40 \mathrm{~km}$ grid spacing both in horizontal and vertical directions. All of the following analyses are restricted to a $936 \times 936 \times 128$ box above the IMaX FOV (unless otherwise stated).

\section{Analysis of the extrapolation results}

\subsection{Solution consistency}

Both the magnetogram and disequilibrium of the initial atmosphere drive the evolution of the system to an MHS state. The disequilibrium of the initial atmosphere is due to nonuniform gas pressure and density in the photosphere. Figure 4 illustrates the compensation of forces in the initial state and final equilibrium. The horizontal component of the forces are shown in panels (a) and (c), while the vertical components are illustrated in panels (b) and (d). In the initial state, we find the residual force is greater than the Lorentz force (see panels (a) and (b)), so that this state is obviously far from a MHS equilibrium. As mentioned before, this promotes further optimization. We see, in the final solution, that the Lorentz force is balanced effectively by the pressure gradient and also vertically by gravity (see panels (c) and (d)). On average, the residual force is $43 \%$ of the Lorentz force in the transverse direction, while the percentage is only $24 \%$ in the vertical direction below $2 \mathrm{Mm}$. These residual forces are not close to 0 . The main contributions come from the photosphere and regions with very high or low $\beta$. The

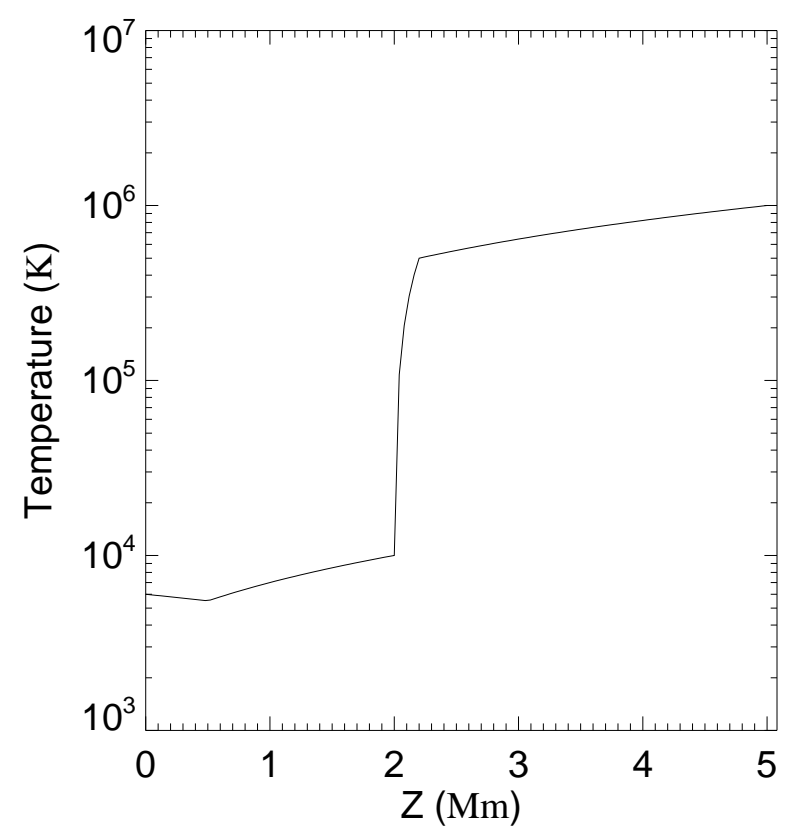

Fig. 3. Temperature profile of the gravity-stratified atmosphere employed as the initial condition.

inadequate boundary condition of plasma, as well as the noise in the measured magnetic field, prevent the MHS extrapolation from balancing forces well on the photosphere. In a very high plasma region $(\beta>10)$ Lorentz forces are too weak to act against plasma forces, which could result in a ratio of residual force over Lorentz force that is much larger than 1 . In a very low plasma region $(\beta<0.1)$ plasma forces are too weak to against Lorentz force, which could result in a ratio that is close to 1 . We recomputed the ratios excluding the bottom boundary and only in regions where $0.1 \geq \beta \leq 10$. The numbers are $23 \%$ and $7.6 \%$ for the transverse and vertical direction, respectively, which means that the major part of the Lorentz force is balanced. Although those two numbers are still not very small, they are acceptable considering that we embedded two data sets into each other (recorded by different instruments and obtained by different inversion technologies, etc.) when producing the magnetogram that provides the bottom boundary. 

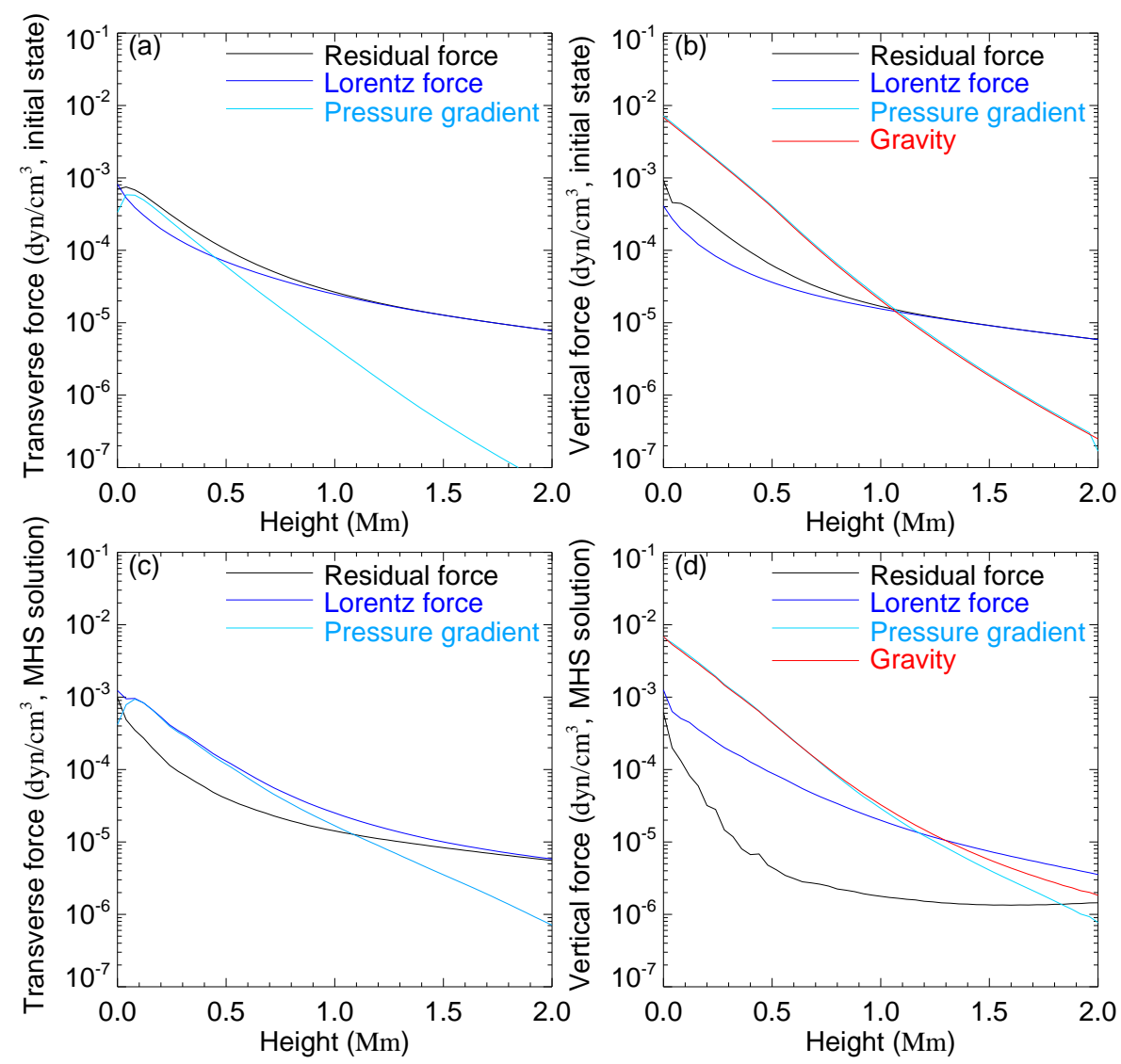

Fig. 4. Planar average of forces as a function of height in the initial unrelaxed state $(a)(b)$ and in the MHS equilibrium $(c)(d)$. Panels $(a)$ and (c) show the transverse direction, while panels $(b)$ and $(d)$ illustrate the forces in the vertical direction.

\subsection{Plasma}

Figure 5 shows plasma distributions from different perspectives. From both top and side views, we see clearly that plasma pressure and density are reduced in strong field regions to keep the force balance. This is consistent with sunspot observations. Similar results were also found both in the LMHS models (Aulanier et al. 1998, 1999; Wiegelmann et al. 2015, 2017) and the previous tests of our MHS code (Zhu \& Wiegelmann 2018, 2019). However, we also find that, at some parts of the active region edges, plasma pressure and density are enhanced. This has never been reported in previous extrapolations. Figure 6 depicts selected Lorentz force vectors in a FOV that is outlined by the red square in Fig. 5(c). We see that the regions with an enhanced gas pressure, which are encircled by blue ellipses, are surrounded with a net inward flux of the Lorentz force. As a result, the plasma is squeezed together. It is also worth noting that the fibril-like plasma pattern traces the magnetic field in Figs. 5(c) and 7(a). Such localized concentrations reflect the nonlinear nature of the MHS system, which cannot be observed in the LMHS model (see Fig. 7).

According to the model of plasma $\beta$ over an active region developed by Gary (2001), the magnetic field dominates plasma above a height of 1-2 Mm (see Fig. 3 in Gary 2001). Figure 8(a) shows that the $\beta_{(z)}$ of the MHS equilibrium is right inside the $\beta$ range illustrated in Gary (2001). A high plasma $\beta$ is a necessary, but is not a sufficient condition for the magnetic field to be non-force-free. To see whether the magnetic field is nonforce-free in the high $\beta$ region, the current-weighted sine of the angle between the magnetic field and the electrical current density (Schrijver et al. 2006) is computed

$\sigma=\sum_{i} \frac{\left|\mathbf{J}_{i} \times \mathbf{B}_{i}\right|}{B_{i}} / \sum J_{i}$.

As shown in Fig. 8(b), $\sigma$ decreases quickly from 0.7 at the photosphere to less than 0.1 above $2.0 \mathrm{Mm}$. Since the effective vertical resolution of the solution is roughly the same as the horizontal resolution of the magnetogram, the high-resolution IMaX data allow us to study this non-force-free layer in detail. Coarser data (e.g., HMI) with a few grid points to resolve this layer meet with difficulties when focusing on the lower atmopshere. Figure 8(c) shows Lorentz force distributions at a height of $0.4 \mathrm{Mm}$. We see strong Lorentz forces are mainly located at edges of strong-field features, where plasma $\beta$ drops precipitous. This is a natural result caused by the strong plasma forces at edges. The great plasma differences at edges of magnetic features in the lower atmosphere are routinely observed.

\subsection{Relation between plasma pressure, current density, and photospheric brightness}

Figure 9 shows the mapping of plasma and current density onto an image acquired by SUNRISE/SuFI. The extrapolation data are cut according to the SuFI FOV of $15^{\prime \prime} \times 38^{\prime \prime}$. Bright points, which are clearly seen in the intergranular lanes in panel (c), are typically regarded as nearly vertical slender flux tubes with kilogauss magnetic fields (Solanki 1993; Nagata et al. 2008; Requerey et al. 2014). The lateral radiative inflow makes the tubes hot and bright (Spruit 1976). Thus the tubes are visible as bright points at wavelengths sensitive to temperature (Schüssler et al. 2003; Riethmüller et al. 2010).

Figures $9 \mathrm{a}$ and $\mathrm{b}$ show that regions of high plasma pressure and strong electric current density coincide. Most of these regions are located near the edges of magnetic flux tubes. These flux tubes, which appear as photospheric bright points at SuFI $3000 \AA$ (see Fig. 9(c)), are often accompanied by high plasma pressure (below $400 \mathrm{~km}$ ) and are often surrounded by electric 

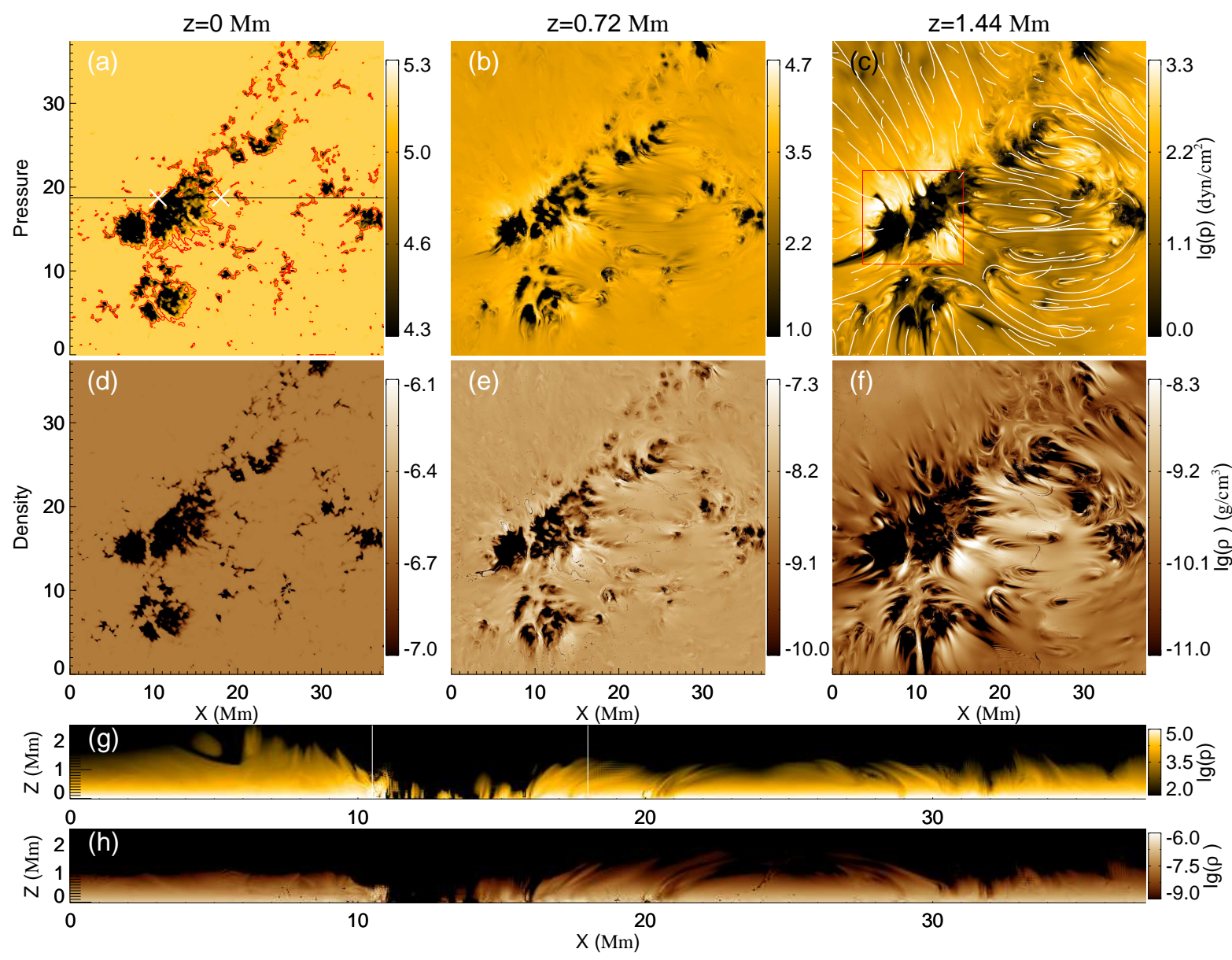

Fig. 5. (a)- $(f)$ Gas pressure and density at heights of $0 \mathrm{Mm}, 0.72 \mathrm{Mm}$, and $1.44 \mathrm{Mm}$. $(g)(h)$ Pressure and density at the plane $y=18.7 \mathrm{Mm}($ black line in panel $(a)$ ). The red contours in panel $(a)$ outline the locations at which magnetic field strength is $1000 \mathrm{G}$. The two white " $\mathrm{X}$ " marks in panel $(a)$ indicate the intersections of the $1000 \mathrm{G}$ contour and the black line. These marks are also the seed points of the two white lines in panel $(g)$. The red rectangle in panel $(c)$ specifies the FOV of Fig. 6. Uniformly selected magnetic field lines in panel $(c)$ range from $600 \mathrm{~km}$ to $1400 \mathrm{~km}$.

current. At the edges of flux tubes, the plasma and magnetic field interact, which leads to the high current and co-spatial high plasma pressure. Such a high current density around magnetic bright points is also reminiscent of the electric current sheets expected to bound flux tubes. It is worth noting that many bright points do not have corresponding enhancements in the electric current. This may be related to the local dynamics. It must also be kept in mind that the MHS model does not take into account the radiation.

\subsection{Comparisons of magnetic fields and chromospheric fibrils}

The SuFI instrument provides diffraction-limited images at $3968 \AA$ with contributions from both the photosphere and lowto-mid-chromosphere (Jafarzadeh et al. 2017). The observed slender fibrils at this wavelength are the dominant structures (see Fig. 10(a)) in the SuFI FOV. It is generally believed that long fibrils in the chromosphere outline magnetic fields in this layer (de la Cruz Rodríguez \& Socas-Navarro 2011; Jing et al. 2011; Schad et al. 2013; Leenaarts et al. 2015; Zhu et al. 2016). We plot field lines within sub-volumes spanning the $600-1400 \mathrm{~km}$ height range, which implies that low field lines $(\leq 600 \mathrm{~km})$ are ignored. Seed points of the field lines are uniformly selected in the photosphere. Figure 10 shows that most fibrils trace field lines nicely. The similar field line patterns of different extrapolation models imply that plasma forces have limited impact on the large-scale magnetic field of this potential-like active region, at least in the height range from 600 to $1400 \mathrm{~km}$.

In order to quantitatively show the degree of agreement between fibrils and field lines, we computed the angle $\theta$ between fibrils and the plane-of-the-sky component of the magnetic field. We did not carry out the computation on every pixel on the image. Instead, to show more clearly the discrepancy among different models, we focused on the regions in which large differences between magnetic vectors of the MHS model and the NLFFF model appear (see contours in Fig. 10). We tried to identify all fibrils in the regions of interest. For each fibril, one point is picked for the statistics. Then we used the method introduced by Inhester et al. (2008) to estimate the orientation of the fibril. The key point of the method is to use the gradient and Hessian matrix of the image intensity to determine the orientation. Then $\theta$ is computed vertically within the 600$1400 \mathrm{~km}$ height range. The smallest $\theta$ is specified as the discrepancy between the fibril and the magnetic vector. For a total of 26 points picked (hence 26 fibrils identified), the averages of $\theta$ for the MHS, NLFFF, and LMHS models are $11.8^{\circ}, 15.7^{\circ}$, and $20.9^{\circ}$, respectively. There are 18 points (nearly $70 \%$ ) at which the $\theta$ of the MHS model is less than those of the other two models. 


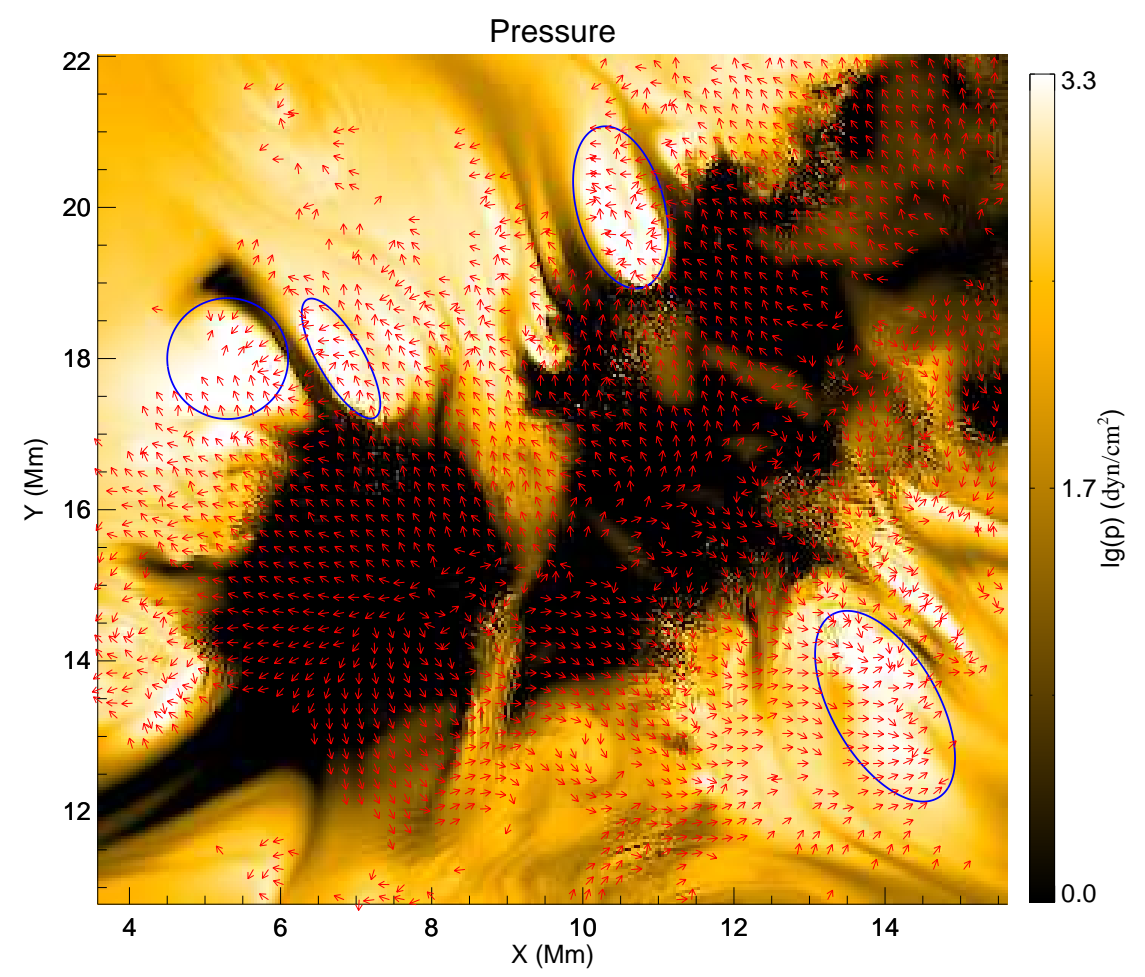

Fig. 6. Two-dimensional Lorentz force vectors overplotted on the gas pressure at a height of $1.44 \mathrm{Mm}$. The plotted FOV is outlined by the red rectangle in Fig. 5(c). Blue ellipses encircle four typical regions where the gas pressure is enhanced.

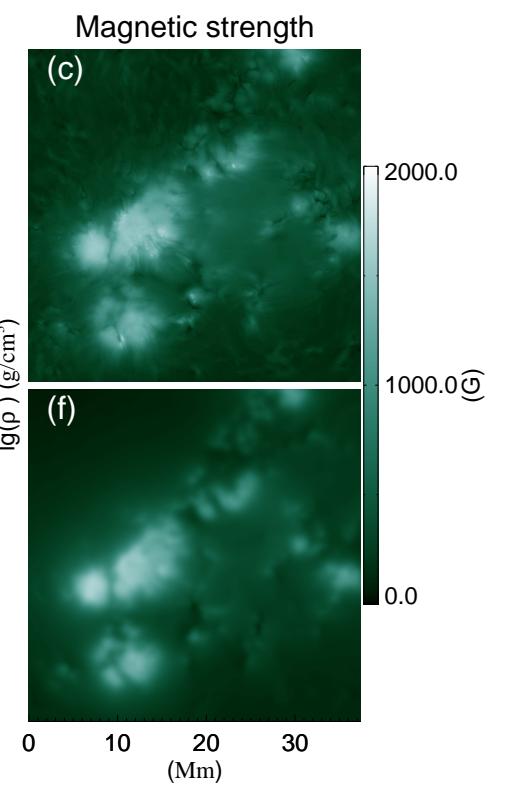

Fig. 7. Pressure, density, and magnetic field strength of the MHS model $(a)(b)(c)$ and the LMHS model $(d)(e)(f)$ at a height of $0.72 \mathrm{Mm}$. Uniformly selected magnetic field lines in panel $(a)$ range from $600 \mathrm{~km}$ to $1400 \mathrm{~km}$.
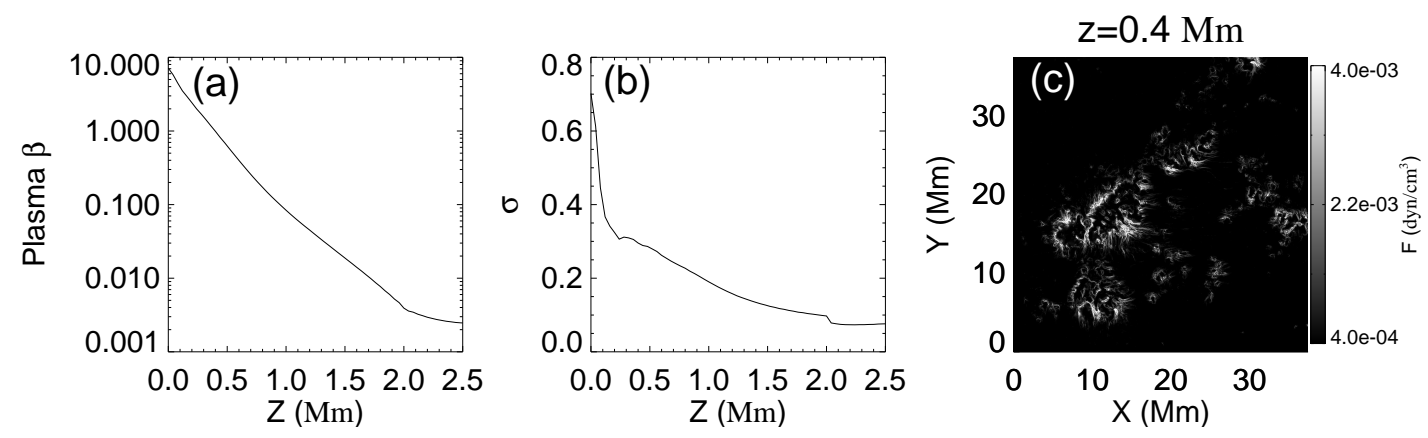

Fig. 8. Planar average of plasma $\beta(a)$ and of current-weighted sine $\sigma(b)$, where $\sigma$ is the angle between the magnetic field and current, as functions of height. (c) The magnitude of Lorentz force at a height of $0.4 \mathrm{Mm}$. 


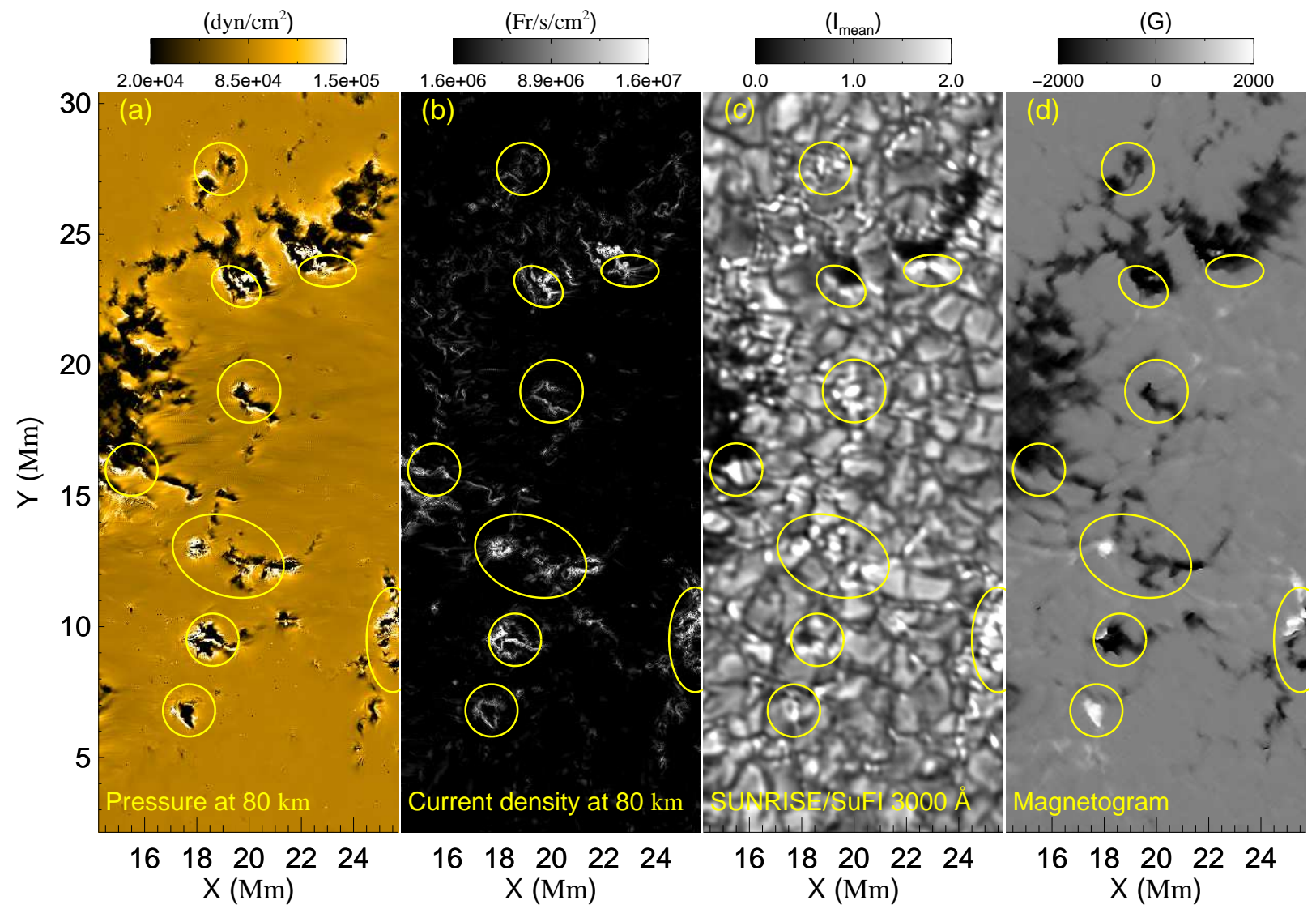

Fig. 9. (a) Gas pressure and (b) current density at height of $80 \mathrm{~km}$. (c) SuFI $3000 \AA$ Amage. (d) IMaX magnetogram. Strong pressure regions are outlined by yellow ellipses.

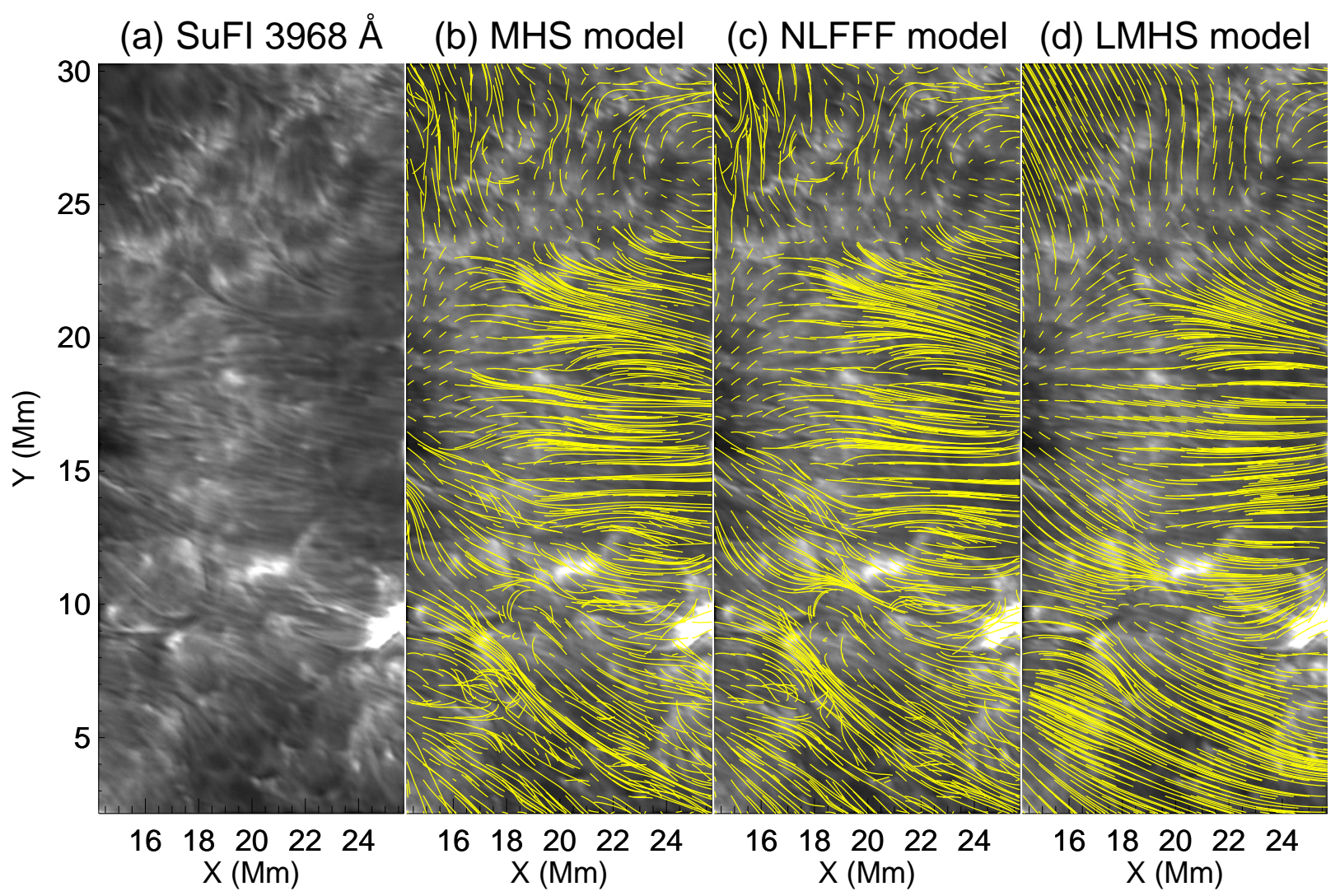

Fig. 10. Magnetic field lines of different models within the heights $[600,1400] \mathrm{km}$ overplotted on the image observed in Ca II H core line with $1.1 \AA$ wide filter. The field lines are rooted in the same set of seed points in all panels. 


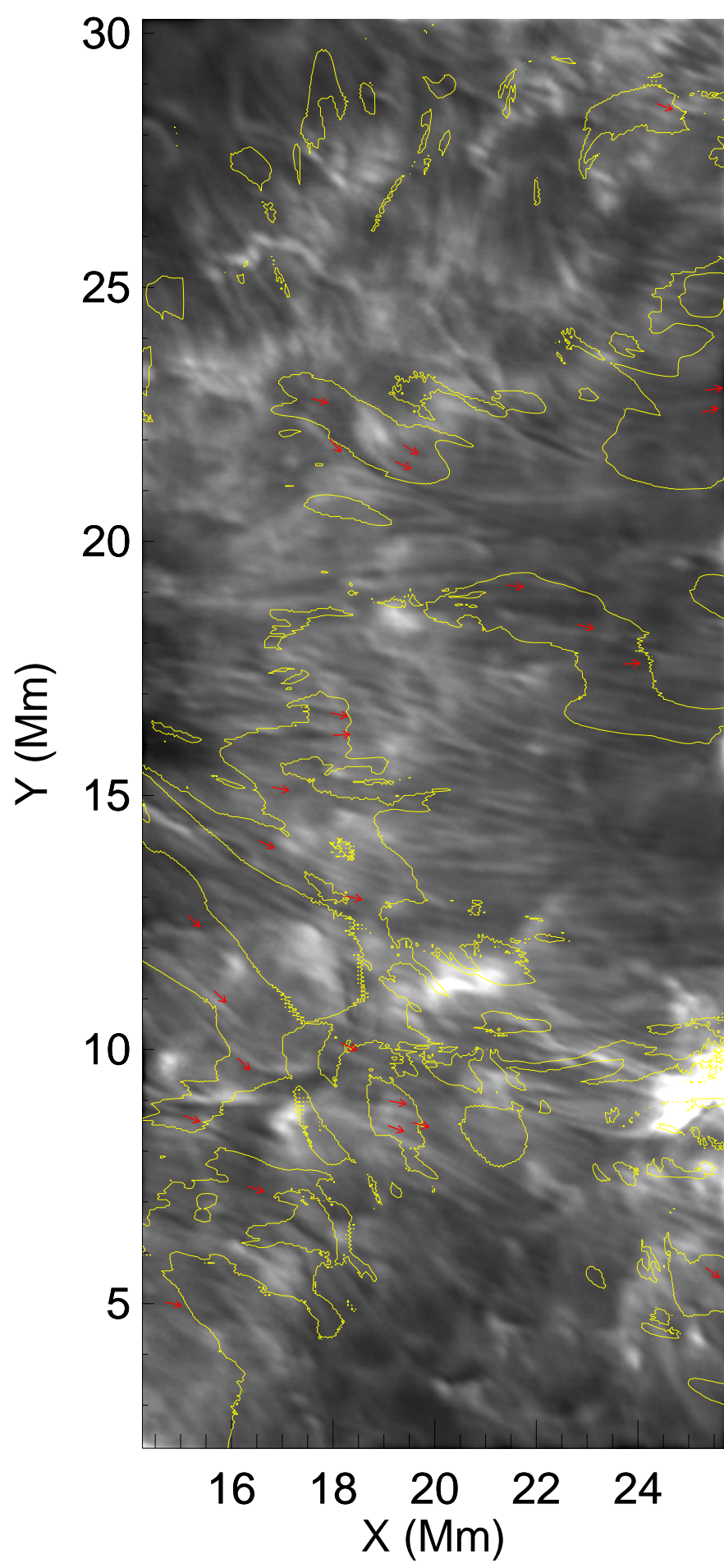

Fig. 11. Contours show where the average of angles between the magnetic vectors of the MHS and NLFFF range from $600 \mathrm{~km}$ to $1400 \mathrm{~km}$ equals $5^{\circ}$. The red vectors depict orientations of the fibrils. Each vector is started from the point at which $\theta$ has the largest value over this fibril.

\section{Conclusions and perspectives}

In this work we apply, for the first time, a nonlinear MHS code to model the solar lower atmosphere starting from a real magnetogram. A combined vector magnetogram to cover the whole active region is used as the boundary input.

An MHS equilibrium is constructed in which Lorentz forces are effectively balanced by plasma forces. The pressure and den- sity depletion take place in the strong field regions together with enhancements in the active region edges. In the low $\beta$ layers, the fibril-like plasma structures clearly outline the magnetic field lines. A thin non-force-free layer is resolved within about 50 grid points in the $\mathrm{z}$ direction. The high plasma pressure and co-spatial high electric current appear around the bright points prominent in SuFI $3000 \AA$ images. Although similar general patterns of the magnetic fields are found in different types of extrapolations (MHS, linear MHS, and nonlinear force-free fields), a quantitative comparison implies that the magnetic field vectors of the MHS model are more aligned with the orientation of the chromospheric fibrils observed at SuFI $3968 \AA$.

As mentioned before, the gas pressure and density are optimized independently. This means the initial isothermal temperature profile cannot be kept. An equation of state is needed to close the MHS equations. However, the present version of the code deals with pressure and density separately. Future studies may focus on the extrapolation under more constrains from observations.

The MHS extrapolation converges at a much lower speed than the NLFFF extrapolation. In this application with $2336 \times$ $1824 \times 128$ grid points, the MHS code runs about $55 \mathrm{hr}$ on 2 Intel Xeon Gold 6150 CPU with 18 cores while the NLFFF code only takes $1 \mathrm{hr}$. Considering the thinness of the nonforce-free layer, a future extrapolation could consist of a timeconsuming MHS model in the lower atmosphere and a much faster NLFFF model in the higher atmosphere by using the model below as the boundary input. Such a combination is a superior solution to the NLFFF model with preprocessed magnetogram (Wiegelmann et al. 2006). Last but not least, the MHS equilibrium, as computed by the new model, can serve as the initial conditions for time-dependent, data-driven MHD simulations (Jiang et al. 2016; Guo et al. 2019).

Acknowledgements. We acknowledge constructive suggestions of the referee and valuable discussions with B. Inhester and T. Riethmüller. The German contribution to SUNRISE and its reflight was funded by the Max Planck Foundation, the Strategic Innovations Fund of the President of the Max Planck Society (MPG), DLR, and private donations by supporting members of the Max Planck Society, which is gratefully acknowledged. This project has also received funding from the European Research Council (ERC) under the European Union's Horizon 2020 research and innovation programme (grant agreement No. 695075) and has been supported by the BK21 plus program through the National Research Foundation (NRF) funded by the Ministry of Education of Korea. This work was also supported by DFG-grant WI 3211/4-1.

\section{References}

Aschwanden, M. J. 2013, Sol. Phys., 287, 323

Aschwanden, M. J. 2016, ApJS, 224, 25

Aulanier, G., Démoulin, P., Schmieder, B., Fang, C., \& Tang, Y. H. 1998, Sol. Phys., 183, 369

Aulanier, G., Démoulin, P., Mein, N., et al. 1999, A\&A, 342, 867

Bagenal, F., \& Gibson, S. 1991, J. Geophys. Res., 96, 17663

Barthol, P., Gandorfer, A., Solanki, S. K., et al. 2011, Sol. Phys., 268, 1

Berkefeld, T., Schmidt, W., Soltau, D., et al. 2011, Sol. Phys., 268, 103

de la Cruz Rodríguez, J., \& Socas-Navarro, H. 2011, A\&A, 527, L8

De Rosa, M. L., Schrijver, C. J., Barnes, G., et al. 2009, ApJ, 696, 1780

Frutiger, C., Solanki, S. K., Fligge, M., \& Bruls, J. H. M. J. 2000, A\&A, 358, 1109

Gandorfer, A., Grauf, B., Barthol, P., et al. 2011, Sol. Phys., 268, 35

Gary, G. A. 2001, Sol. Phys., 203, 71

Gent, F. A., Fedun, V., Mumford, S. J., \& Erdélyi, R. 2013, MNRAS, 435, 689

Gilchrist, S. A., \& Wheatland, M. S. 2013, Sol. Phys., 282, 283

Gilchrist, S. A., Braun, D. C., \& Barnes, G. 2016, Sol. Phys., 291, 3583

Guo, Y., Xia, C., Keppens, R., Ding, M. D., \& Chen, P. F. 2019, ApJ, 870, L21

Inhester, B., Feng, L., \& Wiegelmann, T. 2008, Sol. Phys., 248, 379

Jafarzadeh, S., Solanki, S. K., Stangalini, M., et al. 2017, ApJS, 229, 10

Jiang, C., Wu, S. T., Feng, X., \& Hu, Q. 2016, Nat. Commun., 7, 11522 
Jiao, L., McClymont, A. N., \& Mikic, Z. 1997, Sol. Phys., 174, 311

Jing, J., Yuan, Y., Reardon, K., et al. 2011, ApJ, 739, 67

Kahil, F., Riethmüller, T. L., \& Solanki, S. K. 2017, ApJS, 229, 12

Lanza, A. F. 2008, A\&A, 487, 1163

Leenaarts, J., Carlsson, M., \& Rouppe van der Voort, L. 2015, ApJ, 802, 136

Low, B. C. 1985, ApJ, 293, 31

Low, B. C. 1991, ApJ, 370, 427

MacTaggart, D., Gregory, S. G., Neukirch, T., \& Donati, J. F. 2016, MNRAS, 456, 767

Malanushenko, A., Schrijver, C. J., DeRosa, M. L., Wheatland, M. S., \& Gilchrist, S. A. 2012, ApJ, 756, 153

Martínez Pillet, V., Del Toro Iniesta, J. C., Álvarez-Herrero, A., et al. 2011, Sol. Phys., 268, 57

McClymont, A. N., \& Mikic, Z. 1994, ApJ, 422, 899

Metcalf, T. R., Leka, K. D., Barnes, G., et al. 2006, Sol. Phys., 237, 267

Miyoshi, T., Kusano, K., \& Inoue, S. 2020, ApJS, 247, 6

Nagata, S., Tsuneta, S., Suematsu, Y., et al. 2008, ApJ, 677, L145

Neukirch, T. 1997, A\&A, 325, 847

Neukirch, T., \& Wiegelmann, T. 2019, Sol. Phys., 294, 171

Osherovich, V. A. 1985, ApJ, 298, 235

Pesnell, W. D., Thompson, B. J., \& Chamberlin, P. C. 2012, Sol. Phys., 275, 3

Petrie, G. J. D. 2000, PhD Thesis, Department of Mathematics and Statistics, University of St. Andrews, North Haugh, St Andrews KY16 9SS

Petrie, G. J. D., \& Neukirch, T. 2000, A\&A, 356, 735

Requerey, I. S., Del Toro Iniesta, J. C., Bellot Rubio, L. R., et al. 2014, ApJ, 789, 6
Riethmüller, T. L., Solanki, S. K., Martínez Pillet, V., et al. 2010, ApJ, 723, L169 Ruan, P., Wiegelmann, T., Inhester, B., et al. 2008, A\&A, 481, 827

Schad, T. A., Penn, M. J., \& Lin, H. 2013, ApJ, 768, 111

Scherrer, P. H., Schou, J., Bush, R. I., et al. 2012, Sol. Phys., 275, 207

Schrijver, C. J., De Rosa, M. L., Metcalf, T. R., et al. 2006, Sol. Phys., 235, 161

Schüssler, M., Shelyag, S., Berdyugina, S., Vögler, A., \& Solanki, S. K. 2003, ApJ, 597, L173

Solanki, S.K. 1987, PhD Thesis, Max Planck Institute for Solar System Research, Justus-von-Liebig-Weg 3, 37351 Göttingen, Germany

Solanki, S. K. 1993, Space Sci. Rev., 63, 1

Solanki, S. K., Riethmüller, T. L., Barthol, P., et al. 2017, ApJS, 229, 2

Spruit, H. C. 1976, Sol. Phys., 50, 269

Wiegelmann, T. 2004, Sol. Phys., 219, 87

Wiegelmann, T., \& Inhester, B. 2003, Sol. Phys., 214, 287

Wiegelmann, T., \& Neukirch, T. 2006, A\&A, 457, 1053

Wiegelmann, T., Inhester, B., \& Sakurai, T. 2006, Sol. Phys., 233, 215

Wiegelmann, T., Neukirch, T., Ruan, P., \& Inhester, B. 2007, A\&A, 475, 701

Wiegelmann, T., Yelles Chaouche, L., Solanki, S. K., \& Lagg, A. 2010, A\&A, 511, A4

Wiegelmann, T., Neukirch, T., Nickeler, D. H., et al. 2015, ApJ, 815, 10

Wiegelmann, T., Neukirch, T., Nickeler, D. H., et al. 2017, ApJS, 229, 18

Zhao, X., \& Hoeksema, J. T. 1993, Sol. Phys., 143, 41

Zhu, X., \& Wiegelmann, T. 2018, ApJ, 866, 130

Zhu, X., \& Wiegelmann, T. 2019, A\&A, 631, A162

Zhu, X. S., Wang, H. N., Du, Z. L., \& Fan, Y. L. 2013, ApJ, 768, 119

Zhu, X., Wang, H., Du, Z., \& He, H. 2016, ApJ, 826, 51 